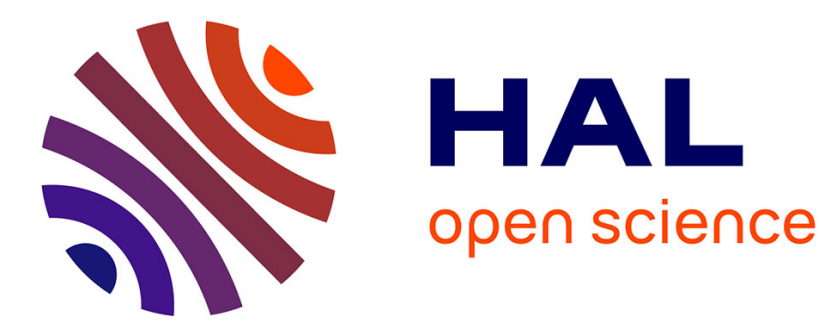

\title{
Le conflit : une motivation à double tranchant pour l'apprenant
}

\author{
Nicolas Sommet, Darnon Céline, Fabrizio Butera
}

\section{To cite this version:}

Nicolas Sommet, Darnon Céline, Fabrizio Butera. Le conflit : une motivation à double tranchant pour l'apprenant. Etienne Bourgeois; Gaëtane Chapelle. Apprendre et faire apprendre, PUF, pp.285-296, 2011, Apprendre, 978-2-13-058391-2. 10.3917/puf.brgeo.2011.01.0285 . hal-01348988

\section{HAL Id: hal-01348988 \\ https://hal.science/hal-01348988}

Submitted on 26 Jul 2016

HAL is a multi-disciplinary open access archive for the deposit and dissemination of scientific research documents, whether they are published or not. The documents may come from teaching and research institutions in France or abroad, or from public or private research centers.

$$
\text { Copyright }
$$

L'archive ouverte pluridisciplinaire HAL, est destinée au dépôt et à la diffusion de documents scientifiques de niveau recherche, publiés ou non, émanant des établissements d'enseignement et de recherche français ou étrangers, des laboratoires publics ou privés. 


\section{Le conflit : une motivation à double tranchant pour l'apprenant}

Nicolas Sommet, Université de Lausanne

Céline Darnon, Clermont Université \& Institut Universitaire de France

Fabrizio Butera, Université de Lausanne

Cédric et Stéphane sont deux étudiants en première année de Psychologie. Lors d'une séance de travaux pratiques, ils cherchent la cause du trouble bipolaire. Cédric avance que l'origine est d'ordre génétique, tandis que Stéphane pense qu'elle est à chercher dans l'environnement social du patient. Que va faire Cédric ? Va-t-il se replonger dans ses cours de psychopathologie pour comprendre l'étiologie du trouble ? Ou va-t-il essayer d'affirmer la supériorité de son opinion sur celle de Stéphane ? Ou va-t-il plus simplement lui donner raison ? Cette situation de confrontation à un autrui en désaccord est qualifiée par certains auteurs de conflit sociocognitif ${ }^{1}$. Ce dernier se caractérise par une double incertitude. D'une part, il remet en question la maitrise de la tâche (le versant cognitif du conflit : « Ma réponse est-elle valide ? »). D’autre part, il peut constituer une menace pour la compétence (le versant social du conflit : «L'autre est-il meilleur que moi ?»).

\section{Les vertus du conflit}

Selon la perspective constructiviste piagétienne, lorsqu'un individu est soumis à une nouvelle information, il tente de l'assimiler dans ses structures cognitives préexistantes. Imaginons par exemple qu'un enfant voit un Appaloosa (cheval de selle américain) pour la première fois. Parce que cet animal partage un certain nombre de caractéristiques communes avec les races chevalines qu'il connaît déjà (p.ex., mammifère domestique, sabot unique, crinière, etc.), l'enfant va l'intégrer à la catégorie « cheval ». Cependant, il arrive parfois

\footnotetext{
${ }^{1}$ Doise, W. \& Mugny, G. (1997). Psychologie sociale et développement cognitif (2ème Edition). Paris: Armand
} Colin. 
qu'une information nouvelle apparaisse incohérente avec les connaissances préalables.

Imaginons cette fois que l'enfant voit un zèbre pour la première fois. Parce que cet animal se différencie des races chevalines qu'il connaît déjà (i.e., présence de rayures verticales noires et blanches), l'enfant ne va pas pouvoir l'intégrer à la catégorie « cheval ». Le processus d'assimilation va échouer. On parle alors de conflit cognitif. Afin de régler ce conflit, l'enfant va devoir accommoder ses structures cognitives en créant une nouvelle catégorie d'animal qu'il appellera sans doute « cheval avec rayures » et, plus tard, lorsqu'il aura appris le terme, « zèbre ». Dans tout processus d'acquisition des connaissances existe une dynamique de conflits et d'ajustements internes.

\section{La régulation du conflit}

Dans les années soixante-dix, W. Doise et G. Mugny constatent que les aspects sociaux des apprentissages ont été négligés dans les travaux de Piaget, alors que la plupart des conflits cognitifs surviennent dans des contextes interindividuels (p.ex., un(e) enseignant(e) et sa classe). Les premières recherches ${ }^{2}$ sur le conflit sociocognitif montrent que les désaccords entre individus prédisent les progrès cognitifs. En favorisant la décentration (la capacité à se mettre à la place de l'autre), l'utilisation de stratégies de raisonnement complexes, ainsi qu'un retour compréhensif sur la tâche et ses propriétés, le conflit sociocognitif apparaît comme un moteur pour le développement cognitif. Selon ces conclusions, Cédric et Stéphane devraient donc bénéficier de leur désaccord.

Cependant, d'autres travaux mettent en évidence des effets moins univoques. Le conflit sociocognitif ne va s'avérer profitable que dans le cas où l'autre est davantage perçu comme un support informationnel que comme une menace pour la compétence ${ }^{3}$. Revenons à

\footnotetext{
2 Doise, W., Mugny, G., \& Perret-Clermont, A.-N. (1975). Social interaction and the development of cognitive operations. European Journal of Social Psychology, 5, 367-383.

3 Darnon, C., Butera, F., \& Mugny, G. (2008). Des conflits pour apprendre. Presses universitaires de Grenoble.
} 
notre exemple. Si Cédric ne se sent pas menacé par Stéphane (par exemple, si leur climat relationnel est coopératif), le fait que son partenaire soit en faveur d'une explication environnementale plutôt que biologique va introduire un doute sur la validité de sa réponse. Centré sur la tâche, Cédric va alors reconsidérer le problème plus en profondeur, en réévaluant la pertinence de chacune des propositions. La régulation du conflit sera dite épistémique, puisque basée sur l'élaboration de la connaissance. Sans doute, Cédric parviendra-t-il alors à intégrer sa réponse à celle de Stéphane. Suite au conflit, il sera plus à même de comprendre les mécanismes de vulnérabilité génétique associés à l'émergence du trouble bipolaire, à savoir qu'il faut être porteur de certains gènes mais que ceux-ci ne s'expriment que dans le cas d'un environnement fragilisant.

À l'inverse, dans le cas où l'autre est davantage perçu comme une menace pour la compétence que comme un support informationnel, le conflit sociocognitif va être délétère pour l'apprentissage. Si Cédric se sent menacé par Stéphane (par exemple, si leur climat relationnel est compétitif), le fait que son partenaire ne soit pas d'accord avec lui va introduire un doute sur sa compétence. Puisqu'il y a divergence, c'est que l'un a raison tandis que l'autre a tort, que l'un est meilleur et l'autre est moins bon. Dans une telle situation conflictuelle, centrée sur la comparaison sociale, Cédric va réagir en défendant et en protégeant sa compétence. La régulation du conflit est alors dite relationnelle.

Plus spécifiquement, il existe pour Cédric deux moyens de réguler le conflit de manière relationnelle. Soit il peut maintenir son point de vue tout en invalidant le point de vue de Stéphane, réaffirmant alors que la cause du trouble bipolaire est héréditaire et non environnementale («J'ai raison, tu as tort. »). Ce comportement d'auto-confirmation se nomme régulation relationnelle compétitive. Soit Cédric peut épouser le point de vue de Stéphane tout en invalidant le sien («Tu as raison, c'est moi qui ai tort. »), s'accordant sur le fait que le trouble bipolaire est d'origine environnementale et non héréditaire, sans plus 
élaborer sur la question. Ce comportement de complaisance se nomme régulation

relationnelle protective. Que Cédric régule le conflit de manière relationnelle compétitive (en résistant à l'influence d'autrui) ou de manière relationnelle protective (en se soumettant à l'influence d'autrui), il sera centré sur la question de la compétence relative et non sur la connaissance per se.

À partir du moment où il est régulé de manière épistémique, le conflit constitue un puissant vecteur de progrès cognitif ${ }^{4}$. Par la confrontation à un point de vue différent, l'individu recourt à des stratégies d'élaboration plus complexes que s'il avait été seul. En revanche, lorsqu'il est régulé de manière relationnelle, le conflit peut entraver l'apprentissage.

\section{Apprendre ou réussir mieux que les autres?}

Quels sont les facteurs qui orientent vers l'une ou l'autre de ces régulations du conflit ? Lorsqu'ils travaillent sur une tâche, les apprenant(e)s peuvent poursuivre différents types de buts : des buts de maîtrise et des buts de performance. Les buts de maîtrise correspondent au désir d'acquérir des compétences, d'apprendre et de progresser. Les buts de performance correspondent au désir de réussir relativement à autrui. Lorsqu'ils poursuivent des buts de maîtrise, les apprenant(e)s sont centré(e)s sur la compréhension de la tâche et tendent à évaluer leur réussite selon un standard absolu («Est-ce que je maîtrise les objectifs liés à la tâche ?»), ou en relation avec leur réussite passée («Est-ce que j’ai progressé sur cette tâche »). En d'autres termes, ils se soucient avant tout de solutionner le problème et d'acquérir des nouvelles compétences. En revanche, lorsqu'ils poursuivent des buts de performance, les apprenant(e)s sont centré(e)s sur la comparaison sociale des compétences et tendent à évaluer leur réussite selon un standard normatif (« Suis-je meilleur(e) ou moins bon(ne) que l'autre ? »). En d'autres termes, ils sont avant tout préoccupés par le fait

\footnotetext{
${ }^{4}$ Buchs C., Darnon C., Quiamzade A., Mugny G., \& Butera F. (2008). Régulation des conflits sociocognitifs et apprentissage. Revue Française de Pédagogie, 163, pp. 105-125.
} 
d'essayer de se montrer compétent(e)s, ou éviter de se montrer incompétent(e)s relativement à autrui $^{5}$.

Supposons que Cédric poursuive des buts de maîtrise. Il ne considérera pas son désaccord avec Stéphane comme une menace pour sa compétence. Au contraire, il percevra son partenaire comme un support informationnel. Cédric sera donc plus à même de réguler le conflit de manière épistémique, intégrant son point de vue à celui de Stéphane. Si en revanche, Cédric poursuit des buts de performance, il considérera son désaccord avec Stéphane comme une menace pour sa compétence. Cédric régulera le conflit de manière relationnelle, soit en se montrant inflexible sur ses positions, soit en se montrant complaisant, et n'aboutissant finalement qu'à la moitié de la compréhension du problème.

Dans une étude que nous avons menée ${ }^{6}$, des participant(e)s étudiaient un texte après avoir reçu une consigne induisant soit un but de maitrise (i.e., il s'agit pour vous d'acquérir de nouvelles connaissances), soit un but de performance (i.e., il s'agit pour vous d'avoir une bonne note au QCM final). Au cours de la tâche, les participant(e)s interagissaient avec un(e) partenaire défendant une position différente. Dans un contexte de buts de maîtrise, plus le conflit augmentait, plus il était régulé de manière épistémique ; dans un contexte de buts de performance, plus le conflit augmentait, plus il était régulé de manière relationnelle. De plus, une autre étude ${ }^{7}$ a montré que dans un contexte de maîtrise, le conflit amenait à un meilleur apprentissage que dans un contexte de buts de performance. Le conflit sociocognitif ne s'avère donc profitable que lorsque les apprenant(e)s sont animé(e)s par une appétence pour la compréhension.

\footnotetext{
${ }^{5}$ Elliot, A. J. (2005). A conceptual history of the achievement goal construct. In A. Elliot \& C. Dweck (Eds.), Handbook of competence and motivation, pp. 52-72. New York: Guilford Press.

${ }^{6}$ Darnon, C., \& Butera, F. (2007). Learning or succeeding? Conflict regulation with mastery or performance goals. Swiss Journal of Psychology, 66, 145-152.

${ }^{7}$ Darnon, C., Butera, F., \& Harackiewicz, J. (2007). Achievement goals in social interactions: Learning within a mastery vs. performance goal. Motivation and Emotion, 31, 61-70.
} 


\section{Vouloir être bon versus éviter d'être mauvais}

Lorsqu'ils travaillent sur une tâche académique, nous avons vu que des apprenant(e)s peuvent poursuivre des buts de maîtrise (désir d'apprendre) ou des buts de performance (désir de réussir relativement à autrui). Plus précisément, il existe deux types de buts de performance : des buts de performance-approche et des buts de performance-évitement. S'ils correspondent tout deux à une préoccupation quant à la démonstration de ses compétences, les buts de performance-approche sont associés au désir de se montrer meilleur(e) qu'autrui (atteindre la compétence normative), tandis que les buts de performance-évitement sont associés au désir de ne pas se montrer moins bon(ne) qu'autrui (évitement de l'incompétence normative). Typiquement, un(e) élève ayant pour objectif de compter parmi les trois meilleur(e)s de la classe poursuivra des buts de performance-approche. En revanche, un(e) élève dont l'objectif serait de « ne pas faire moins que la moyenne » poursuivra des buts de performance-évitement.

Nos travaux ${ }^{8}$ ont montré qu'une orientation vers des buts de performance-approche prédisait une régulation relationnelle compétitive du conflit (basée sur l'affirmation de son point de vue et l'invalidation de celui de l'autre). Si Cédric a pour objectif d'être meilleur que Stéphane, il restera probablement campé sur sa position en ignorant les propos de son partenaire. Par ailleurs, une orientation vers des buts de performance-évitement prédit une régulation relationnelle protective (basée sur l'affirmation du point de vue de l'autre et l'invalidation du sien). Si Cédric redoute d'être moins compétent que Stéphane, il se montrera probablement complaisant à son égard. Dans les deux cas, Cédric ne fera aucun progrès cognitif.

\section{Les paradoxes de l'expertise}

\footnotetext{
${ }^{8}$ Darnon, C., Sommet, N., Butera, F., Mugny, G., Quiamzade, A., Pulfrey, C., \& Dompnier, B. Performanceapproach and performance-avoidance goals in social interaction: Toward the distinction between two modes of relational conflict regulation. Manuscript en préparation.
} 
Jusqu'à présent, nous ne nous sommes intéressés qu'aux situations conflictuelles entre individus de statuts identiques. Or, les contextes d'apprentissage constituent avant tout des contextes sociaux et l'ensemble des acteurs du monde scolaire, académique ou professionnel ne partage que rarement un statut équivalent. Particulièrement à l'école, les élèves d'une classe sont organisé(e)s selon une hiérarchie (notes, classement). Face à ses étudiant(e)s, un enseignant(e) fait également figure d'autorité légitime, jouissant d'un pouvoir d'expertise. Or, si Vygotsky affirme que les progrès cognitifs ne sont possibles qu'en « résolvant des problèmes sous la guidance d'un adulte ou en collaboration avec un pair plus capable (p. 86) $\gg^{9}$, nous allons voir que ces asymétries de statut peuvent constituer un frein à l'apprentissage, notamment lorsque la compétence de l'autre est perçue comme menaçante.

Revenons une nouvelle fois à notre exemple. Imaginons que Cédric soit un élève moyen et Stéphane l'un des premiers de la classe. Imaginons également que l'enseignant(e) ait insufflé un but compétitif à la tâche, en proposant que les meilleures réponses individuelles rapportent des points supplémentaires au prochain examen. Dans un tel contexte de menace pour la compétence, Cédric souffrant d'un statut inférieur, il est probable qu'il craigne de s'opposer au point de vue de Stéphane et régule le conflit de manière protective, en épousant le point de vue de son partenaire. Par ailleurs, Stéphane, afin d'affirmer la supériorité de son statut, régulera certainement le conflit de manière compétitive.

Dans une étude menée par A. Quiamzade et ses collègues ${ }^{10}$, des participant(e)s étaient confronté(e)s à une réponse divergente émanant d'un(e) partenaire de haut niveau d'expertise. La compétence de l'autre était rendue soit menaçante (par l'existence d'un climat compétitif), soit non menaçante (climat neutre). Dans un contexte non menaçant, les participant(e)s régulaient le conflit de manière épistémique, profitant de l'expertise du

9 Vygotsky, L. S. (1978). Mind in society. Cambridge, MA: Harvard University Press.

10 Quiamzade A., Tomei A., \& Butera F. (2000). Informational dependence and informational constraint: Social comparison and social influences in an anagram task. International Review of Social Psychology, 13, 123150 . 
partenaire. Dans un contexte compétitif et menaçant en revanche, confronté(e)s à un(e) partenaire de plus haut statut, les participant(e)s régulaient le conflit d'une manière relationnelle protective en se soumettant au jugement de leur partenaire. En d'autres termes, ils adoptaient un comportement de complaisance exempt de toute élaboration cognitive. Délétère pour l'apprentissage, cette dernière situation est notamment à éviter lorsqu'elle concerne les membres du corps professoral (dits experts) face à leurs élèves (dits novices). À partir du moment où l'expertise de l'enseignant(e) est perçue comme menaçante, les élèves sont plus susceptibles de se montrer complaisants à son égard et très statiques face aux apprentissages. Or, à l'école, l'élève ne doit pas rester un simple réceptacle des savoirs. Il est bien plus utile qu'il critique les informations qu'il reçoit, ce qui l'aide à les comprendre et plus ou moins les intégrer. S'il est menacé dans ses compétences, il adopte le point de vue de l'enseignant(e) sans critiquer, sans comprendre. Ce n'est que lorsqu'il perçoit l'enseignant(e) comme un support informationnel profitable qu'il est capable d'apprentissage réel.

\section{Des conflits pour apprendre}

L'apprentissage est une perpétuelle remise en question. En mathématique, apprendre les décimales, c'est remettre en question le fait qu'un nombre impair ne soit pas divisible par deux. En biologie, apprendre les théories néo-darwinistes, c'est remettre en question nos préconceptions transformistes naïves pour expliquer la marche de l'évolution. À une plus grande échelle, la connaissance scientifique et philosophique s'est construite sur une série de conflits devenus célèbres, entre Platon et Aristote, entre les newtoniens et les cartésiens. Les conflits sont indispensables à tout progrès cognitif. Ils nous permettent pas à pas, d'échafauder nos connaissances, d'appréhender et de (re)construire le réel.

Toutefois, le conflit perd ses bénéfices dès lors que l'autre est davantage perçu comme une menace que comme un support informationnel, lorsque l'apprenant(e) poursuit 
des buts de performance plutôt que de maîtrise par exemple, ou lorsque le différentiel de statut est menaçant. Selon leurs pratiques pédagogiques, les enseignant(e)s peuvent créer un climat de classe plus ou moins propice à une régulation épistémique des conflits. Quatre éléments interviennent de façon déterminante dans la régulation du conflit en classe : (1) la représentation du conflit; (2) la position de l'enseignant(e) « expert(e)»; (3) l'évaluation de l'élève ; (4) le travail en groupe.

La représentation du conflit. Culturellement, les élèves perçoivent souvent le conflit comme étant mauvais. Ils l'associent à une tension relationnelle et tendraient donc à l'éviter. De la même manière, confronté(e) à la réponse conflictuelle d'un(e) élève, un(e) enseignant(e) peut parfois chercher à minimiser le désaccord et «arrondir les angles ». Or, nous l'avons vu, l'évitement du conflit est néfaste pour l'apprentissage. Changer cette représentation du conflit en le présentant comme une aide potentielle plutôt qu'un obstacle permettrait de réduire ce risque. Quelle attitude convient-il alors d'avoir dans une telle situation? Lors d'un désaccord, une attitude centrée sur l'évitement du conflit (p.ex.

«Chacun peut voir les choses comme il veut ») ou sur l'affirmation de ses compétences (p.ex. « Tu n'as rien compris. Laisse-moi te réexpliquer. ») ne favorisera pas l'apprentissage. En revanche, certaines études ${ }^{11}$ montrent que privilégier une attitude centrée sur la tâche lors d'un désaccord (p.ex. «Explique-moi ce qui te fait penser cela. ») favorisera la régulation épistémique du conflit et l'apprentissage. Plus généralement, un(e) enseignant(e) gagnerait donc à générer un climat de classe où la démarche critique et la confrontation des points de vues sont valorisées. Certains procédés pédagogiques encourageant la discussion, tels l'organisation de débats ou de projets de groupe, pourraient notamment permettre de revaloriser la représentation du conflit sociocognitif.

\footnotetext{
${ }^{11}$ Darnon, C., Buchs, C., \& Butera, F. (2002). Epistemic and relational conflict in sharing identical vs. complementary information during cooperative learning. Swiss Journal of Psychology, 61, 139-151.
} 
La position de l'enseignant(e) « expert(e) ».Dans le contexte de classe, nous en avons parlé, l'enseignant(e) jouit d'un pouvoir d'expertise sur ses élèves, disposant des connaissances à transmettre. S'il(elle) représente un indéniable support informationnel, l'enseignant(e) peut également constituer une menace pour les compétences des élèves, leur rappelant leur propre « incompétence ». (p. ex. «C'est faux », ou « Tu n'as rien compris »). Or, tout l'enjeu est que ces derniers ne se représentent pas cette asymétrie de compétence comme étant menaçante. Si, par exemple, l'élève se sent contraint d'avoir un point de vue similaire à l'enseignant(e), alors ceci va l'amener à se focaliser sur la peur de donner une réponse qui ne sera pas validée par l'enseignant(e) et à se montrer complaisant. L'information transmise ne sera traitée que superficiellement. Toutefois, si dans cette même situation, la menace en provenance de l'enseignant(e) est évacuée, alors l'élève pourra profiter de son expertise. L’information transmise sera alors élaborée de manière durable. Finalement, les enseignements remettent souvent en cause les préconstruits épistémiques des apprenant(e)s ; il est donc essentiel que les informations conflictuelles soient intégrées et traitées en profondeur. Un(e) enseignant(e) stimulant la participation orale, acceptant la contradiction et tolérant l'erreur parviendrait sans doute à réduire la menace associée à son statut et faire en sorte de stimuler une démarche constructiviste dans l'apprentissage.

L'évaluation des élèves. Nombre d'instances éducatives demeurent ancrées dans un système compétitif. Les pratiques d'évaluations notamment y favorisent l'émergence de buts de performance. Classements, notes, menace du redoublement sont autant de facteurs susceptibles de motiver l'apprenant(e) à vouloir dépasser(ou éviter d'être dépassé(e) par) les autres plutôt que se dépasser soi-même. Lorsqu'en France nous assistons à une augmentation du taux d'obtention du baccalauréat (plus de 85\% en 2009), nombre d'individus en déduisent que le diplôme n'en a que moins de valeur. En effet, élèves et parents d'élèves ont pour usage 
de penser que pour être brillant à l'école, il faut réussir là ou les autres échouent. L'idéologie de la compétition et du mérite, via les politiques de numerus clausus ou des évaluations publiques plutôt que privée (p.ex. remise des copies en classes de façon dégressive) favorisent une conception du savoir et des compétences comme étant négativement interdépendants (si l'autre réussit, ma réussite n'en est que moins estimable). L'autre est plus souvent perçu comme un concurrent potentiel que comme un support informationnel. Des évaluations critériées (validation de compétences), privées (p.ex.. pas de classement, pas de report de la moyenne de classe) ainsi que la récompense de l'effort serviraient la régulation épistémique des conflits et l'apprentissage. Pour que cet objectif soit atteint, néanmoins, il ne suffit pas de compter sur la bonne volonté de certains enseignant(e)s éclairé(e)s. Il convient de s'efforcer de rappeler que la fonction première des structures éducative est de former l'élève et non de l'évaluer en vue de sa sélection.

Le travail en groupe. Nous avons vu que la compétence de l'autre pouvait représenter une menace. Lors d'un travail en dyade ou en groupe, les différences de statut (p.ex. compétence perçue de l'autre via les notes), peuvent exacerber cette menace et avoir une influence néfaste sur les apprentissages. Spécifiquement, lors d'un travail collectif, les recherches ${ }^{12}$ montrent que les élèves souffrant d'un statut défavorisé ont moins d'influence sur les autres membres du groupe, interagissent moins et progressent moins. Afin que l'apprentissage collectif soit effectif, il convient donc de diminuer les disparités perçues de statut. Ainsi, régulés de manière épistémique, les conflits dans le groupe serviraient-ils les apprentissages. Une méthode pour réduire la menace peut consister à partager des ressources au sein du groupe. Des études ${ }^{13}$ ont mis en évidence le fait que, lorsqu'ils disposaient

12 Cohen, E. G. (1994). Restructuring the classroom: Conditions for productive small groups. Review of Educational Research, 64, 1-35.

13 Buchs, C. \& Butera, F. (2009). Is a Partner's competence threatening during dyadic cooperative work? It depends on resource interdependence. European Journal of Psychology of Education, 24, 145-154. 
d'informations complémentaires plutôt qu'identiques, les membres du groupe apprenaient de manière plus efficiente(voir aussi le chapitre de C. Buchs, I. Gilles et F. Butera dans cet ouvrage). Supposons par exemple qu'un groupe d'élèves doive préparer un exposé sur la seconde guerre mondiale. Il peut-être judicieux de leur assigner à chacun un objectif particulier : l'un devra travailler sur la montée du fascisme en Europe, tandis qu'un autre travaillera sur la résistance et un dernier sur le rôle de l’URSS. Pour accéder à la compréhension globale du problème, chaque élève devra compter sur l'autre. Les différences de statuts seront alors minimisées, chacun(e) des apprenant(e)s jouissant d'une expertise sur sa thématique particulière. Globalement, ce type de modus operandi favorisera le partage des informations, chacun contribuant au groupe de façon homogène, la confrontation des points de vue catalysant alors les progrès épistémiques.

Les conflits sont indispensables aux progrès cognitifs. Néanmoins, ils perdent leurs bénéfices dès lors qu'un(e) partenaire est perçu(e) comme une menace. Pour prévenir cela, l'enseignant(e) est à même de mettre en place un climat de classe encourageant la régulation épistémique des conflits. Se garder d'insuffler une logique compétitive entre les élèves et prévenir la menace associée aux différences de statut par la coopération sont autant de méthodes qui vont favoriser les apprentissages. Epicure disait déjà du conflit que « celui qui est vaincu a gagné davantage, à proportion de ce qu'il vient d'apprendre ». Plutôt que le mérite et l'affirmation de soi, ce sont ces valeurs que l'école gagnerait à propager. 